\title{
Polymorphous low grade adenocarcinoma of hard plate
}

\author{
Metin $\mathrm{M}^{1}$, Dogan $\mathrm{S}^{2}$, Yazici $\mathrm{H}^{3}$, Soy $\mathrm{FK}^{4}$, Dilci $\mathrm{A}^{5}$, Avcu $\mathrm{M}^{1}$ and Fidan $\mathrm{V}^{*}$ \\ ${ }^{1}$ Department of ENT, Ahi Evran Univ, Turkey \\ ${ }^{2}$ Department of ENT, Adiyaman Univ, Turkey \\ ${ }^{3}$ Department of ENT, Balıkesir Univ, Turkey \\ ${ }^{4}$ Department of ENT, Mardin Gov Hosp, Turkey \\ ${ }^{5}$ Department of ENT, Eskisehir Yunus Emre Gov Hosp, Turkey
}

\begin{abstract}
Polymorphous low-grade adenocarcinoma (PLGA) is a rare malignancy which could usually be seen in minor salivary glands. The incidence of PGLA in all minor salivary gland tumor are $10 \%$ and $25 \%$ in all malignant minor salivary gland tumor. Salivary gland adenocarcinomas have been known as aggressive however progress of PLGA is silent and non-aggressive. Pathologic diagnosis of this tumor is so difficult because of multiple cytological variations. Besides this, treatment options and prognosis of disease is not clear and controversial. In this case diagnosis, management and treatment of PLGA of hard plate is presented with recent literature review.
\end{abstract}

\section{Introduction}

Polymorphous low grade adenocarcinoma (PLGA) is a malignant tumor that usually develops in the minor salivary glands [1]. PLGA is $10 \%$ of all minor salivary gland tumors and $25 \%$ of minor salivary gland malignancies [2]. Although many salivary gland adenocarcinomas are known to be aggressive, this type of salivary gland adenocarcinoma is quite silent. PLGA was first reported by Batsakis et al. in 1983 and described as 'terminal duct carcinoma'. In 1984, Evans and Batsakis published 14 additional adenocarcinomas of the same type as terminal duct carcinoma and described them as PLGA. Pathological diagnosis of this tumor is quite difficult although tumor consists of different malignant cell types [3]. However; diagnosis, management and prognosis of this tumor cases are still controversial. Detailed studies should be applied focusing this issue for enlightening the etiology and management of this tumor. In this case, we presented diagnosis, management and treatment approach of palatal PLGA case and literature review about this rare tumor.

\section{Case report}

Informed consent was obtained from patient before planning of this study. A fifty-five year old male patient was admitted to our clinic with complaint of upper palate swelling (Figure 1). Routine ENT history, physical examination and laboratory studies are normal. As a result of the CT imaging, approximately $2 \times 1.5 \mathrm{~cm}$ mass lesion starting from the hard palate and extending to the soft palate was reported. Periostal invasion was not present according to the CT. Pathological lymphadenopathy was not detected with physical examination and neck USG. The patient was operated with $1 \mathrm{~cm}$ safety surgical margin and mass lesion was resected ( Figure 2). The defect was reconstructed with island flap pedicled by bilateral second molar tooth (Figure 3). In histopathological examination; the tumor has irregular borders and mucinous salivary glands were observed close to tumor in tissue sample (Figure 4). Tumor was consisted of solid, atypical microcystic and cribriform structures with atypical, pleomorphic, epithelial cells. Immunohistochemistrical examination showed diffuse / strong staining with Vimentin (Figure 4B ) and S-100 (Figure 4C), focal / weak staining with CD117 ( Figure 4D) and Ki-67 proliferation index of $1-2 \%$ in tumor cells. PLGA was diagnosed according to low grade atypia of cells, diffuse / strong staining with vimentin and S-100, focal / weak positive with CD117 and low score of Ki-67 proliferation index. No recurrences were detected at postoperative 6 month follow-up.

\section{Discussion}

PLGA is a very rare tumor, usually affecting the minor salivary glands of the oral cavity. PLGA is frequently located in the palate (49$78 \%$ ), followed by buccal mucosa and upper lip, which can be seen in the oral cavity, lower lip, alveolar arc, and minor salivary glands of tongue $[1,4]$. PLGA is the second most common malignancy following mucoepidermoid carcinoma of minor salivary glands [5,6]. It has been accepted that PLGA is only observed in the minor salivary glands however PLGA cases has also been reported that in the major salivary glands [5,7], in the paranasal sinuses [8], in the breasts [9] and in the vulva and vagina $[10,11]$ in recent years. PLGA tumors can be observed in lungs as primary or metastatic lesions $[12,13,14]$. Same histological and immunohistochemical characteristics are observed in different located PLGA tumors as in minor salivary glands [5]. It is often seen in 6th and 7th decades although could be observed in adolescent period [15]. It is commonly seen in female patients, female to male ratio is $2,4: 1$ $[16,17]$. In the study of Pittsburgh University with 24 subjects; mean of age is 59,4 . 14 patients had palate, 3 patients had nasopharyngeal, 3 patients had floor of mouth, 2 patients had upper lip, 1 patient had tonsil, 1 patient had parotid PGLA tumor in that study [3]. In our patient, the lesion was located at the palate, most common site.

Correspondence to: Associate Professor Vural Fidan MD, Department of ENT, Eskisehir Yunus Emre Gov Hosp, Turkey, E-mail: vuralf@mynet.com

Key words: Polymorphous lowgrade adenocarcinoma, hard palate, salivary gland Received: January 06, 2017; Accepted: January 19, 2018; Published: January 23 , 2018 
Histologically, PLGA has no capsules and has infiltrative tumor borders. Different groups of cells with variable growth patterns are observed. These different cell groups usually consist of a group of cells arranged in tubular, solid, papillary, microcytic, cribriform or fascicular pattern. Since the histological structure of PLGA shows similar features in pleomorphic adenoma, adenoid cystic carcinoma and low grade papillary adenocarcinoma, these tumors should be considered in differential diagnosis $[1,3,4,16]$.

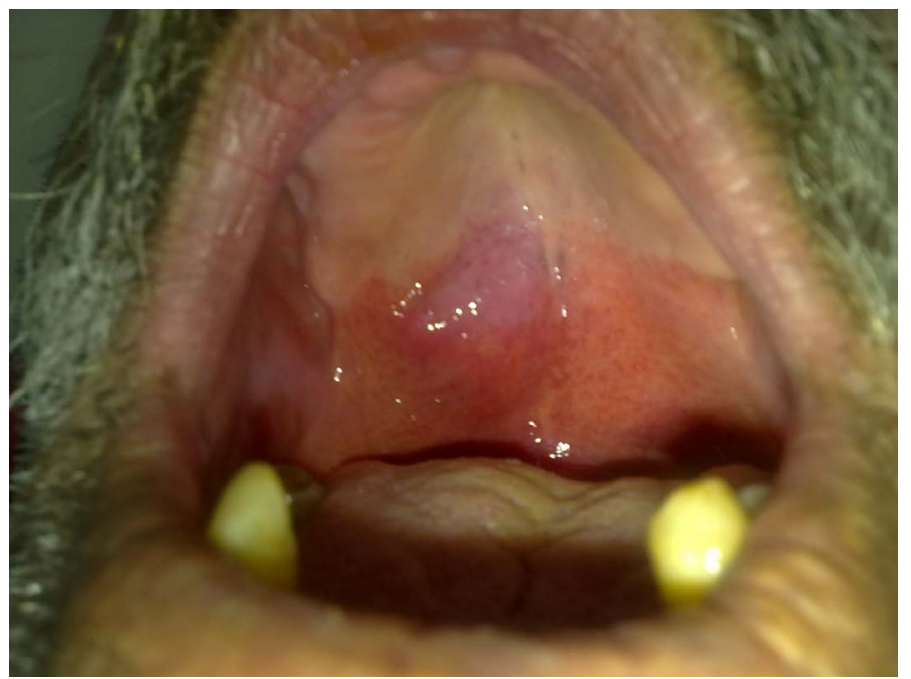

Figure 1. Preoperative image of the tumor

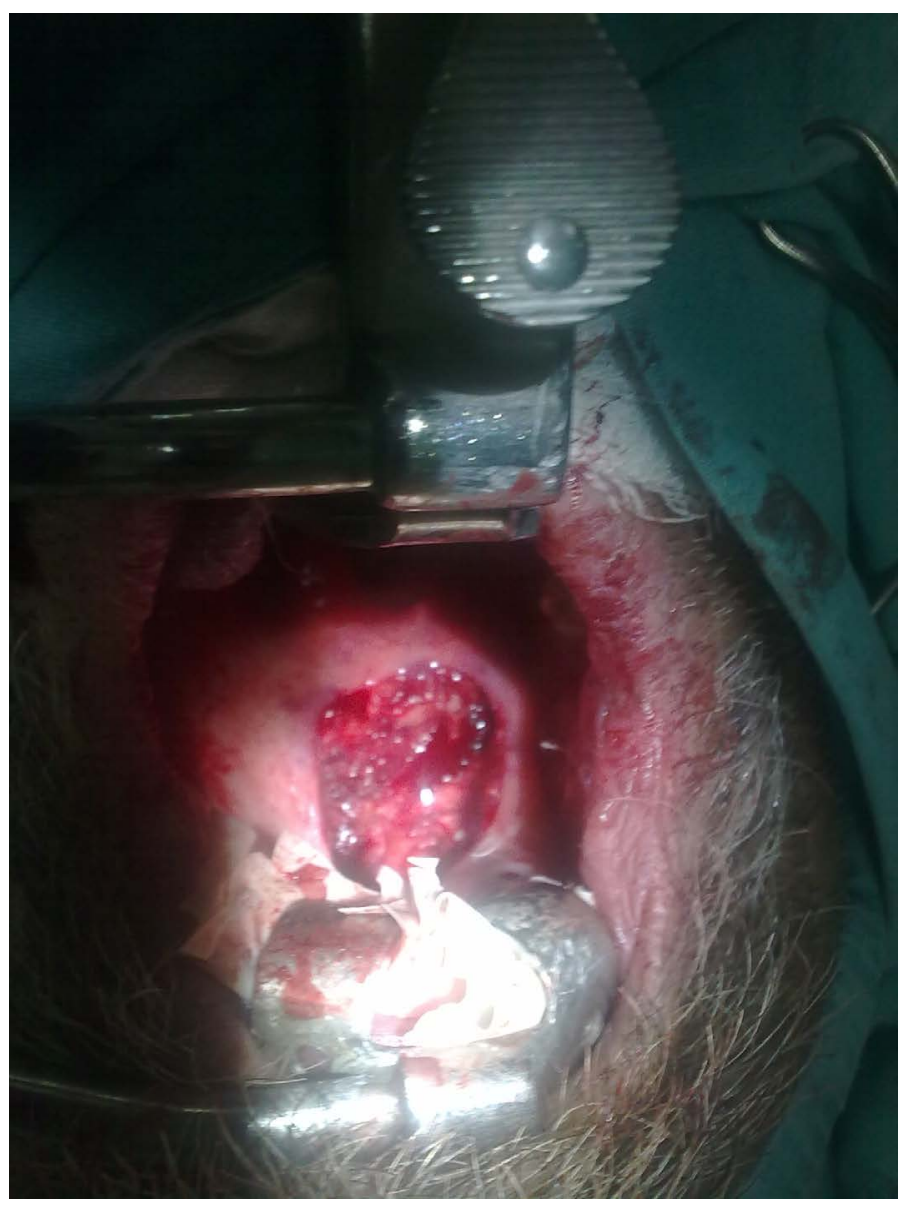

Figure 2. Image of the defect after excision of tumor

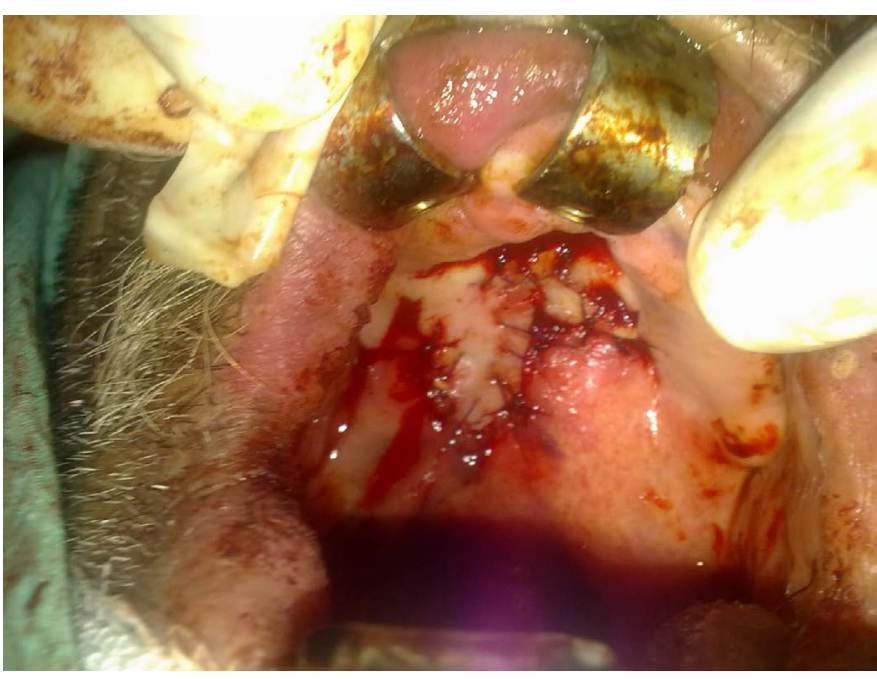

Figure 3. Image of the reconstruction of the defect with island flep

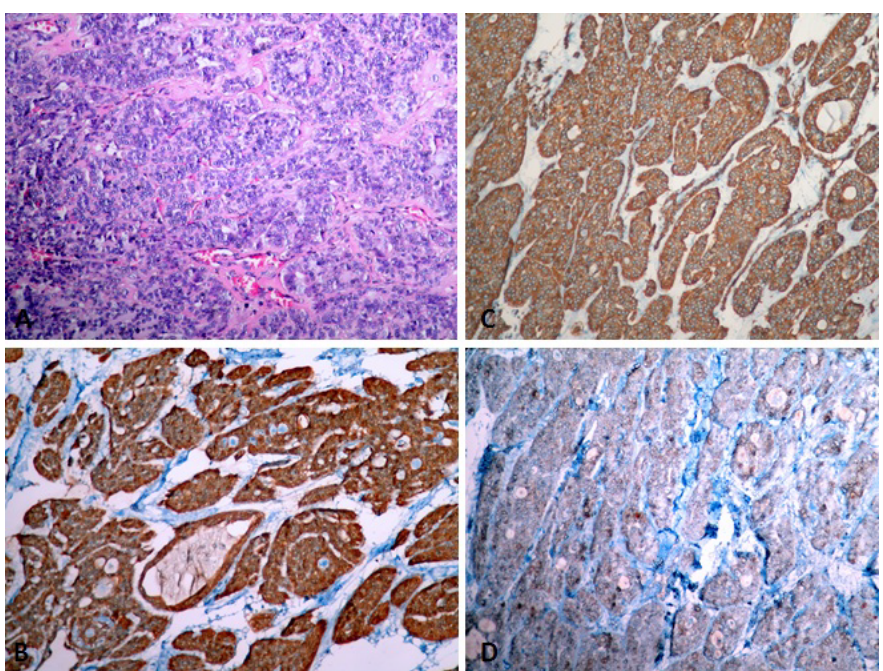

Figure 4. Histopathological apperance of tumor tissue

This may be difficult to diagnose PLGA due to morphological heterogenity and may be confused with tumors such as pleomorphic adenoma and adenoid cystic carcinoma, and immunohistochemical studies may be required. Another important point to note is the possibility that these tumors can be transformed into high grade malignancies over time [3]. The presence of a papillary cystic pattern in the PGLA cases is thought to correlate with aggressive clinical progression [5]. According to some authors, papillary cystic pattern should be classified in a different class [3]. PLGA is accepted as a noninvasive tumor despite local infiltrative and perineural, perivascular invasive features [2,5]. Nodal metastasis and distant metastasis are rarely seen $[14,15]$. Local agressive tumors are also be described in literature $[3,14,15]$. Positive surgical margin, recurrent surgeries, previous radiotherapy, diagnosis in early ages are risk factors for aggressive clinical progression $[3,5,15]$. The most appropriate treatment approach for PLGA is wide limited local excision [1-5]. Postoperative radiotherapy is recommended only in cases where the tumor is seen at the surgical margin. In the presence of cervical metastases, adjuvant radiotherapy and radical surgical excision are recommended. Radical surgery is recommended in recurrent cases [1-4]. A limited number of cases require modified radical neck dissection. It has been reported that the tumor may recurrence after locally excision through follow up 
[3]. Combination of surgery with radiotherapy provides limited benefit, and little information is available on the effectivity of chemotherapy treatment [5]. Many authors have stated that PGLA can be fatal in long-term follow-up. In a study involving 40 patients, patients were reported to have been living for a long time, but $12.5 \%$ of the 10 year mortality rate [18]. In this case, tumor was resected with wide limited local excision and reconstructed with island flap of bilateral second molar tooth pedicled. Pathologic examination of the patient revealed no papillary cystic pattern or neck metastasis. Surgical margins were intact therefore radiotherapy and neck dissection were not done. The patient was followed up taking into consideration the possibility of recurrence or late metastasis.

\section{Conclusion}

PGLA is rare and newly defined malignancy and pathological diagnosis is also quite challanging. Recurrence and late metastasis could be observed during follow up. Mortality and morbidity are high in case of recurrence or metastasis. Therefore follow up of these patients is very important during clinical practice. More detailed studies and case reports focusing this issue can increase the knowledge of clinicians so management and treatment of these tumors can improved.

\section{References}

1. Gupta S, Kumar CA, Raghav N (2011) Polymorphous low-grade adenocarcinoma of the palate: report of a case. Indian J Cancer 48: 518-519.

2. Batsakis JG, Pinkston GR, Luna MA (1983) Adenocarcinoma of the oral cavity: a clinicopathologic study of terminal duct carcinoma. J Laryngol Otol 97: 825-835

3. Seethala RR, Johnson JT, Barnes EL, Myers EN (2010) Polymorphous low-grade adenocarcinoma: The University of Pittsburgh experience. Arch Otolaryngol Head Neck Surg 136: 385-392

4. Khosla D, Verma S, Gupta N, Punia RS, Kaur G, Pandey AK, et al. (2017) Polymorphous low grade adenocarcinoma of the parotid in a teenager. Iran $J$ Otorhinolaryngol 29: 299-302.

5. Olusanya AA, Akadiri OA, Akinmoladun VI, Adeyemi BF (2011) Polymorphous low grade adenocarcinoma: literature review and report of lower lip lesion with suspected lung metastasis. J Maxillofac Oral Surg 10: 60-63.
6. Waldron CA, el-Mofty SK, Gnepp DR (1988) Tumors of the intra oral minor salivary glands: a demographic and histologic study of 426 cases. Oral Surg Oral Med Oral Pathol 66: 323-333.

7. Yih WY, Kratochvil FJ, Stewart JC (2005) Intraoral minor salivary gland neoplasms: review of 213 cases. J Oral Maxillofacial Surg 63: 805-810.

8. Charous DD, Cunnane MF, Rosen MR (2005) Recurrent polymorphous low-grade adeno carcinoma manifesting as a sinonasal mass: a case report. Ear Nose Throat $J$ 84: 354357.

9. Asioli S, Marucci G, Ficarra G (2006) Polymorphous adenocarcinoma of the breast. Report of three cases. Virchows Arch 448: 29-34

10. Madhura MG, Kumar BV, Suma S, Sarita Y (2016) Cribriform adenocarcinoma of minor salivary gland: A mimic of polymorphous low-gradeadenocarcinoma. $J$ Oral Maxillofac Pathol 20: 536-539.

11. Young S, Leon M, Talerman A (2003) Polymorphous low-grade adenocarcinoma of the vulva and vagina: a tumor resembling adenoid cystic carcinoma. Int J SurgPathol 11: 43-49.

12. Cho KD, Jung HJ, Cho DG (2007) Primary polymorphous low grade adenocarcinoma of lung treated by sleeve bronchial resection: a case report. J Korean Med Sci 22: 373376 .

13. Hannen EJ, Bulten J, Festen J (2000) Polymorphous low grade adenocarcinoma with distant metastases and deletions on chromosome 6q23-q ter and 11q23-q ter: a casereport. J Clin Pathol 53: 942-945.

14. Larbcharoensub N, Sanpaphant S, Witoonpanich P, Tuntiyatorn L, Tungkeeratichai J, et al. (2016) Polymorphous low-grade adenocarcinoma of the epiglottis: A case report. Mol Clin Oncol 4: 779-782.

15. Lee VK, McCaughan BC, Scolyer RA(2004) Polymorphous low-grade adenocarcinoma in the lung: a case report. Int J SurgPathol 12: 287-292.

16. Hunter JB, Smith RV, Brandwein-Gensler M (2008) Low-Grade PapillaryAdenocarcinoma of the Palate: The Significance of distinguishing it from Polymorphous Low-Grade Adenocarcinoma. Head and Neck Pathol J 2: 316-323.

17. Toida M, Shimokawa K, Makita H (2005) Intra oral minor salivary gland tumours: a clinicopathological study of 82 cases. Int J Oral Maxillofac Surg 34: 528-532.

18. Harry L, Evans MD, Mario A (2000) Polymorphous low-grade adenocarcinoma a study of 40 cases with long-term follow up and an evaluation of the importance of papillary areas. Am J SurgPathol 24: 1319-1328.

Copyright: (C2018 Metin M. This is an open-access article distributed under the terms of the Creative Commons Attribution License, which permits unrestricted use, distribution, and reproduction in any medium, provided the original author and source are credited. 